\title{
Image Recovery By Peaceman-Rachford Operator Splitting Method
}

\author{
Yubao Sun \\ Institute of computer science and technology \\ Nanjing University of science and technology \\ Nanjing, China
}

\author{
Zhihui Wei \\ Institute of computer science and technology \\ Nanjing University of science and technology \\ Nanjing, China
}

\begin{abstract}
In the solution of image recovery inverse problem, the objective functional can often be modeled as the minimization of the sum of two (not necessarily differential)lower semicontinuous convex functional in a real Hilbert space. A decomposition method derived from Peaceman-Rachford splitting algorithm for monotone operator-splitting is proposed to solve this generic problem. Applications to image deblurring and non-Gaussian denoising in a tight frame are also presented. Finally numerical computations comparing with other classical methods are demonstrated.
\end{abstract}

Keywords-component; image recovery; style; convex variational; operator splitting ; Peaceman-Rachford; DouglasRachford

\section{INTRODUCTION}

The objective of an image recovery problem is to estimate the original image from the observation images which are physically related to it in terms of some known degradation models. This wide class of inverse problems encompasses problems such as image reconstruction, image deblurring, and image denoising. Mathematically, image recovery problems are most commonly formulated as variational problem, the resulting criterion can often related to Bayesian Maximal a Posterior (MAP) estimation, integrating the prior knowledge and the consistence with the observation image. The objective is often to minimize the sum of two convex functions in real Hilbert space $H$

$$
\text { Problem } P_{0}: \min _{x \in H} f(x)+g(x)
$$

Where $f: H \rightarrow(-\infty,+\infty)$ and $g: H \rightarrow R$ are in class $\Gamma_{0}(H)$ of proper lower semi-continuous convex function. In this variational framework, neither of the functions need be differentiable, thus capture broad range of image restoration problem. The only additional request is that at least one should be finite, which is a wild restriction.

The monotone operators splitting methods provide a powerful method for solving this specific convex variational problem. The chief advantage of a splitting method is able to transform original problem into a sequence of much easier sub-problems which involves only the function $f$ or only $g$.Monotone operator splitting method can essentially be categorized into four principal classes: forward-backward splitting (in short FBS), Peaceman-Rachford splitting [1] (in short PRS), and Douglas-Rachford [1, 2] (in short DRS), and the little used double backward class. Recently, splitting algorithm for image recovery has received much attention. FBS algorithm has been proposed to solve problem numerically, but requiring that $f$ or $g$ is differentiable with a Lipschitz continuous gradient [3]. Many state of the art algorithms [4] can be contained in this general framework. In practice, objective functional may be differentiable with a non-Lipschitz gradient or not non-differentiable at all. Thus In [5], a DRS algorithm was introduced to relax the assumption of differentiability of $f$ and $g$. In fact, PRS method were originally formed to solve differential equations in the special linear case [6].Then the method was extended to the nonlinear problem of finding a zero of the sum of two maximal monotone operators in Hilbert space in [1].For general maximal monotone operators, Lions and Mercier proved that it is unconditionally stable. From the numerical result, it seems that PRS requires roughly half the computation of DPS in parabolic problem. For the hyperbolic problem, PRS seems to be more accurate than DPS. Given that DRS scheme is only first order accurate in time, we cannot expect the solution to be highly accurate. However, the PRS scheme is second order accurate in both time and space, thus it is likely to have a more accurate solution.

In the present paper, we propose an algorithm for solving generic problem $P_{0}$ based on the Peaceman-Rachford operator splitting method. In problem $P_{0}$, neither of the functions needs to be differentiable and, thereby, a broad class of signal recovery problems is captured. At the same time, applications to image deburring and non-Gaussian denoising with a tight frame are also demonstrated.

\section{PROPOSED ALGORITHM}

\section{A. Prelimiimries}

In this section, we provide the necessary background on monotone operators, proximal operators. Throughout this paper, $H$ is real Hilbert space with identity operator $I d$, scalar product $<., .>$, and norm $\|\cdot\|$.The definitions and propositions below mainly follow those in convex analysis $[7,8]$.

A set-valued operator $T: H \rightarrow 2^{H}$ is monotone if 
$<x-x^{\prime}, y-y^{\prime}>\geq 0$, Whenever $y \in T(x)$ and $y^{\prime} \in T\left(x^{\prime}\right)$.It is said to be maximal if there is no monotone operator whose graph strictly contains the graph of $T$. The subdifferential operator $\partial f: H \rightarrow 2^{H}$ of a function $f: H \rightarrow(-\infty,+\infty)$ is that

$$
\partial f(x)=\{y \in H \mid f(x+d) \geq f(x)+<d, y>, \forall d \in h\}, \forall x \in H
$$

Proposition 1: If $f \in \Gamma_{0}(H)$, then the subdifferential $\partial f$ is maximal monotone operators on $H$.

Given positive scalar $\gamma$ the proximal operator of $f \in \Gamma_{0}(H)$ is $\operatorname{prox}_{\gamma f}: H \rightarrow H$,

$$
x \mapsto \arg \min _{y \in H} \frac{1}{2 \gamma}\|y-x\|_{2}^{2}+f(y)
$$

For any operator $T$ and positive scalar $\gamma$, the operator $J_{T}^{\lambda}=(I d+\gamma T)^{-1}$ is called a resolvent operator of $T$.-

Proposition 2: Let $f \in \Gamma_{0}(H)$, Then $\operatorname{prox}_{\gamma f}=J_{\partial f}^{\gamma}$.

\section{B. Our algorithms}

Let us consider the following fundamental problem of maximal monotone operator $T$ :

$$
\text { Find } x \in H \text { such that } 0 \in T(x)
$$

The zeroe points of a monotone operator are precisely the fixed points of its resolvents. Thus, the proximal point algorithm for solving this problem generates a sequence $\left\{x^{k}\right\}$ by the iteration:

$$
x^{k+1}=J_{T}^{\gamma}\left(x^{k}\right)
$$

In some cases, $T$ can be expressed as the sum of two maximal monotone operators $T=T_{1}+T_{2}$. Thus Splitting algorithms are designed to solve

$$
0 \in T_{1}(x)+T_{2}(x)
$$

In such way, their resolvents $J_{T_{1}}^{\gamma}$ and $J_{T_{2}}^{\gamma}$ are much easier to evaluate than the resolvent $J_{T}^{\gamma}$ of the whole $T$ in each iteration. In particular, the PRS algorithm studied by Lions and Mercier generates a sequence $\left\{u^{k}\right\}$ by the recurrence

$$
u^{k+1}=\left(2 J_{T_{1}}^{\gamma}-I d\right)\left(2 J_{T_{2}}^{\gamma}-I d\right) u^{k}
$$

Solving problem $P_{0}$ is equivalent to finding a zero point of $\partial[f+g]$.A straightforward way to apply splitting algorithm to $P_{0}$ is to write $\partial[f+g]=\partial f+\partial g$, set $T_{1}=\partial f$ and $T_{2}=\partial g$, and then apply a splitting method to $T_{1}$ and $T_{2}$. Then Proposition 1 asserts that $T_{1}$ and $T_{2}$ are maximal monotone. Moreover, using Proposition 2, we derive at once from (7), the following proximal iteration for solving Problem $P_{0}$

$$
u^{k+1}=\left(2 \operatorname{prox}_{\gamma f}-I d\right)\left(2 \operatorname{prox}_{\gamma g}-I d\right) u^{k}
$$

Then $\left\{u^{k}\right\}$ converges weakly to some point $u \in H$ for a suitable value on the parameter $\gamma$ and $\operatorname{prox}_{\gamma g} u$ is a solution to problem $P_{0}$.An important feature of Algorithm (8) is that it proceeds by splitting in the sense that the proximal operators of $f$ and $g$ are used in separate steps, which makes it particularly attractive in comparison with standard proximal point algorithm, thus decrease the complexity of computation rapidly.

\section{Application To IMAge DBLURRING AND Non- GAUSSIAN IMAGE DENOISING}

As discussed in Section II, the proposed approach is applicable to a wide array of signal recovery problems. In this section, the focus is placed on image deblurring and denoising in the presence of laplacian noise.

We consider the linear inverse problem of recovering image $x$ of size $N_{1} \times N_{2}$ in finite dimensional Euclidean spaces $R^{N}$ from an observation model

$$
y=K x+v
$$

Where $K: R^{N} \rightarrow R^{N}$ is a bounded linear operator and $v \in R^{N}$ is the realization of an additive noise perturbation.

Linear inverse problems are usually ill-conditioned; regularization theory can be adopted to solve them. Certain regularization properties of the ideal signal $x$ are most suitably expressed in terms of the coefficients $\alpha=\left\{\xi_{i}\right\}_{\mathbf{I}=\{1 \leq i \leq I\}}$ of its representation

$$
x=\sum_{i=1}^{I} \xi_{i} \phi_{i}
$$

with respect to a set of atoms $\left\{\phi_{i}\right\}_{i \in \mathbf{I}}$ in Hilbert space. Traditionally such linear representations have almost been focused on orthonormal bases such as Fouries, wavelet. Recently interests have been switched towards more general, redundant representations known as frames. Recall that a set of vectors $\left\{\phi_{i}\right\}_{i \in \mathbf{I}}$ in $H$ constitutes a frame if there exist two constants $\mu$ and $v$ in $(0,+\infty)$ such that

$$
(\forall x \in H) \mu\|x\|^{2} \leq \sum_{i=1}^{I} \xi_{i} \phi_{i} \leq v\|x\|^{2}
$$

the associated frame operator is the injective bounded linear operator

$$
U^{*}: H \rightarrow l^{2}(\mathbf{I}): x \mapsto\left(\left\langle x \mid \phi_{i}\right\rangle\right)_{i \in \mathbf{I}}
$$

The adjoint of which is the surjective bounded linear operator.

$$
U: l^{2}(\mathbf{I}) \rightarrow H:\left(\xi_{i}\right)_{i \in \mathbf{I}} \mapsto \sum_{i \in \mathbf{I}} \xi_{i} \phi_{i}
$$

When $\mu=v$ in (11), $\left\{\phi_{i}\right\}_{i \in \mathbf{I}}$ is said to be a tight frame. For a tight frame $U^{*} U=\eta I d$ and $\eta>0$ is the frame bound .In terms of image representation in frame, (9) can be written as

$$
y=K U \alpha+v
$$

Further,- we- assume- that- the- underlying-solution- $x$ has- $\mathrm{a}^{-}$sparse- $^{-}$representation/approximation- underframe $\left\{\phi_{i}\right\}_{i \in \mathbf{I}}$. Thus according to the observation model-(14)-inthe-frame-domain,-we-would-like-to-find-a-sparse-solution; our strategy will be to infer an estimate of the sparse frame 
coefficient vector $\alpha$ in $R^{I}$ through the Bayesian maximum a posteriori approach.

When operator $K$ is a blur operation, the linear inverse problem corresponds to image deblurring. Assuming a laplacian prior distribution of $\alpha, P(\alpha) \propto \exp \left(-\beta\|\alpha\|_{1}\right)$ to promote a sparse representation in frame, and additive zeromean Gaussian white noise $v$ with known variance, the Bayesian-MAP estimation leads deblurring task to optimization problem:

$$
\hat{\alpha}=\arg \min _{\alpha \in R^{I}} \frac{1}{2}\|y-K U \alpha\|_{2}^{2}+\lambda\|\alpha\|_{1}
$$

Moreover, when operator $K$ is an identical mapping, the linear inverse problem corresponds to image denoising. In this situation, consider independent zero-mean laplacian additive noise and a laplacian prior distribution of $\alpha$. Similar to the above, Bayesian MAP estimation, leads to the following objective functional:

$$
\hat{\alpha}=\arg \min _{\alpha \in R^{I}} \frac{1}{2}\|y-U \alpha\|_{1}+\lambda\|\alpha\|_{1}
$$

Finally, $\lambda>0$ is the regularization parameter. And the restoration results of (15) and (16) are obtained by $\hat{x}=U \hat{\alpha}$.Apparently both problem are special case of problem $P_{0}$, we shall then estimate $\hat{\alpha}$ via the proposed algorithm (8). Let us denote the first term of (15) and (16) as function $f$ and the second as $g$ respectively. It will be necessary to determine the proximity operators of function $f$ and $g$ according to the algorithm (8).

For functions $f$ in (15), the current iterate is $\alpha^{k}$ and the proximal operator of it $\operatorname{prox}_{\gamma_{k} f}\left(\alpha^{k}\right)$ has the expression:

$$
\arg \min _{\alpha \in R^{I}} \frac{1}{2}\|y-K U \alpha\|_{2}^{2}+\frac{1}{2 \gamma_{k}}\left\|\alpha-\alpha^{k}\right\|_{2}^{2}
$$

Zeroing the derivate of this objective function leads to the linear system

$$
\left(\frac{1}{\eta} U^{*} K^{T} K U+\frac{I}{\gamma_{k}}\right) \alpha=\frac{1}{\eta} U^{*} K^{T} y+\frac{\alpha^{k}}{\gamma_{k}}
$$

Because of the regularization term $I / \gamma_{k}$, the coefficient matrix is positive definite. The Conjugate gradient method can be used to solve it. Convergence can be improved using preconditioning techniques.

Following the result in [5], the proximal operator of $f$ in (16) can be determined in a closed form below (Componentwise). Let $U \alpha^{k}=\kappa$,

$$
\operatorname{prox}_{\gamma_{k} f}\left(\alpha^{k}\right)=\alpha^{k}+\eta^{-1} U^{*}\left(y+\operatorname{soft}\left(\kappa-y, \lambda \gamma_{k} \eta\right)-I\right) U\left(\alpha^{k}\right)
$$

The proximal operator of function $g$ in (15) and (16) corresponds to

$$
\operatorname{prox}_{\gamma_{k} g}\left(\alpha^{k}\right)=\operatorname{soft}\left(\alpha^{k}, \gamma_{k} \lambda\right)=\operatorname{sign}\left(\alpha^{k}\right) \max \left(\alpha^{k}-\gamma_{k} \lambda, 0\right)
$$

Which is a component-wise soft threshold shrinkage.
In practice, we need to choose a proper start point for the iteration. In this paper, the start point $\alpha^{0}$ is set as:

$$
\alpha^{0}=U^{*} K^{T} y
$$

In the image denoising case, $K^{T}=I d$.

\section{NUMERICAL SIMULATION}

In this section we present two simulation examples of problem of (15) and (16) solved by our algorithm (8) and compares it with Douglas-Rachford operator splitting algorithm [5]. Test images of size $256 \times 256$ are used and adopts the peak signal noise ratio (PSNR) as a measure of discrepancy between two images $x_{1}$ and $x_{2}$.

\section{A. Image Deblurring}

In this experiment we use the undownsampled wavelet transform, using Daubechies' 4-taps filter, and 1 layers of resolution, leading to a redundancy factor of 4: 1 .and consider a $19 \times 19$ blur kernel with values being $1 /\left(i^{2}+j^{2}+1\right)$ for $-9 \leq i, j \leq 9$, normalized to have a unit sum. The blurred image to noise ratio(BSNR) is $30 \mathrm{~dB}$.Numerical result are shown in Fig.2.In comparison with DPS method, our method improves about $0.9 \mathrm{~dB}$ with $\gamma_{k} \equiv 30$ and $\lambda=0.075$ after only six time iteration.

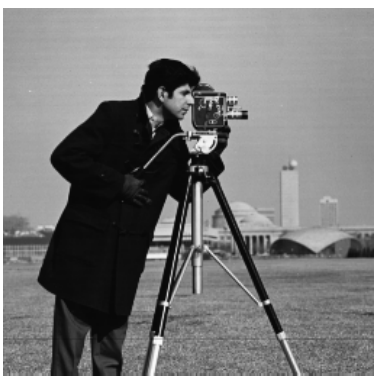

(a) Original cameraman image

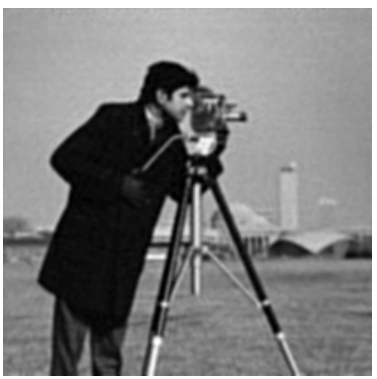

(c) Recovered by DRS $\mathrm{PSNR}=28.2 \mathrm{~dB}$

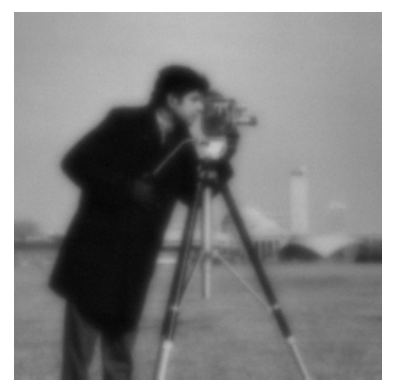

(b) A blur and noise image BSNR: 40dB PSNR: 20.8dB

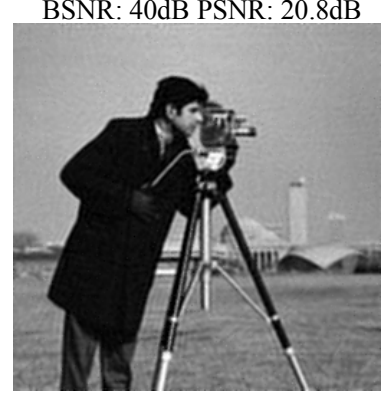

(d) Recovered by PRS $\mathrm{PSNR}=29.1 \mathrm{~dB}$
Figure 1. The performance comparisons between PRS and DRS method for image deblurring

\section{B. Image Denoising with Laplacian Noise}

In this experiment adopt Curvelet [9] as the tight frame and the standard variance of laplacian noise is 15.In this situation, the objective function is not smooth. Numerical results of (16) 
with $\gamma_{k} \equiv 50$ and $\lambda=0.15$ is presented in Fig.2. In comparison with DPS method, our method improves $1.1 \mathrm{~dB}$. The relative ratio of objective functional $\left(f\left(x^{k}\right)+g\left(x^{k}\right)\right) /\left(f\left(x^{0}\right)+g\left(x^{0}\right)\right)$

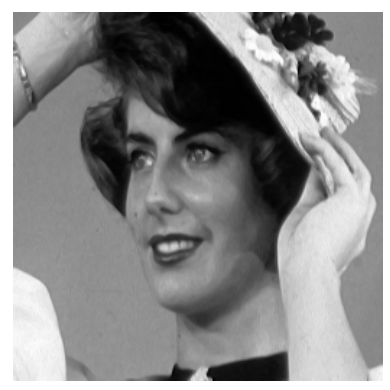

(a)Original image

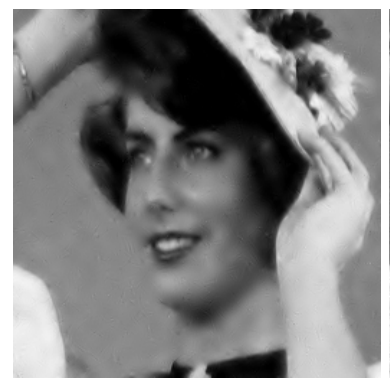

(c) Recovered by DRS PSNR: 29.1dB

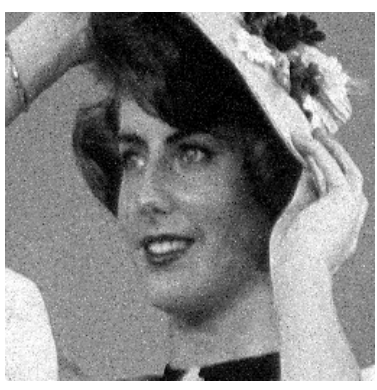

(b) noise image, $\mathrm{PSNR}=23.8 \mathrm{~dB}$

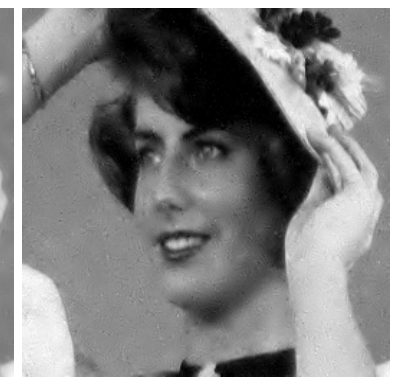

(d) Recovered by PRS PSNR: $30.2 \mathrm{~dB}$
Figure 2. The performance comparisons between PRS and DRS method for image denoising with additive laplacian noise.

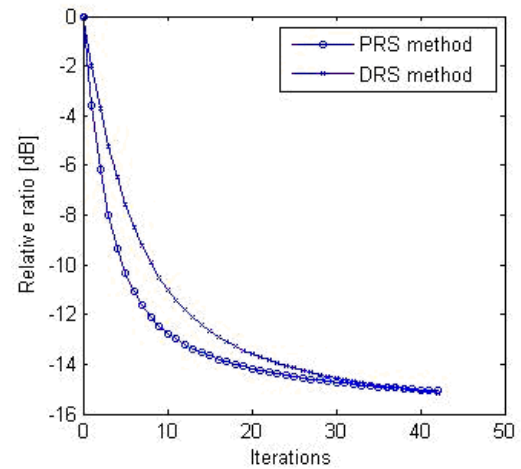

Figure 3. The relative ratio in $\mathrm{dB}$ versus the iteration index $\mathrm{k}$ for $\mathrm{DRS}$ and PRS method. along the iterations are plotted in Fig. 3, showing the good asymptotic convergence behavior of Algorithm (10) and converges faster than DRS method.

\section{CONCLUSION}

In this paper, we have proposed a new algorithm based on PRS method to solve generic convex variational image recovery problem. In this variational framework, the whole objective should be not smooth, thus a broad class of image recovery problems can be encompassed. Numerical simulations demonstrate the effectiveness of our algorithm.

\section{ACKNOWLEDGMENT}

This work is supported by the Nation High-Tech Research and Development Plan of China under Grant No.2007AA12Z142; the National Nature Science Foundation of China under Grant No.60802039,60672074; the Doctoral Foundation of Ministry of Education of China under Grant No.20070288050.

\section{REFERENCES}

[1] P. L. Lions and B. Mercier, "Splitting algorithms for the sum of two nonlinear operators," SIAM J. Numer. Anal, vol. 16, pp. 964-979, December 1979.

[2] J. Eckstein and D. P. Bertsekas, "On the Douglas-Rachford splitting method and the proximal point algorithm for maximal monotone operators," Math. Progr., vol. 55, pp. 293-318, 1992.

[3] P. L. Combettes and V. R. Wajs, "Signal recovery by proximal forwardbackward splitting," Multiscale Model. Simul., vol. 4, no. 4, pp. 11681200, November 2005.

[4] I. Daubechies, M. Defrise, and C. De Mol, "An iterative thresholding algorithm for linear inverse problems with a sparsity constraint," Comm. Pure Appl. Math., vol. 57, pp. 1413-1457, 2004.

[5] P. L. Combettes, J.C. Pesquet, "A Douglas-Rachford splitting approach to nonsmooth convex variational signal recovery," IEEE Journal of Selected Topics in Signal Processing, vol. 1, no. 4, pp.564-574, December 2007.

[6] J. Douglas and H.H. Rachford, "on the numerical solution of the heat conduction problem in two or three space variables," Trans. Amer. Math. Soc., vol.82, pp. 421-439, 1956.

[7] R.T. Rockafellar, Convex Analysis, Princeton, NJ: Princeton University, 1970.

[8] R. S. VARGA, Matrix iterative analysis,2nd ed. New York:SpringerVerlag, 2000..

[9] E. J. Candès and D. L. Donoho, "Recovering edges in ill-posed inverse problems: Optimality of curvelet frames," Ann. Statist., vol. 30, no. 3, pp.784-842, 2002. 